\title{
Numerical simulation study on collinear EFP from warhead with double layer liners penetrating into target
}

\author{
Fangyu Zhang ${ }^{1}$, Changxiao Zhao ${ }^{2}$, Weitao Feng ${ }^{3}$, Chong $\mathrm{Ji}^{4}$, Sunlang Lin ${ }^{5}$, Xin Wang ${ }^{6}$ \\ $1,2,4,5,6$ College of Field Engineering, Army Engineering University of PLA, Nanjing, China \\ ${ }^{3}$ Special Operation College of PLA, Guilin, China \\ ${ }^{2}$ Corresponding author

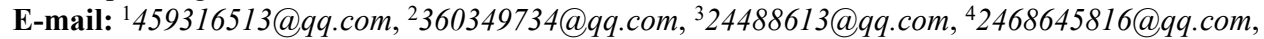 \\ 558243911@qq.com,6310433804@qq.com
}

Received 15 September 2021; received in revised form 27 September 2021; accepted 7 October 2021 DOI https://doi.org/10.21595/vp.2021.22214

Check for updates

Copyright $(2021$ Fangyu Zhang, et al. This is an open access article distributed under the Creative Commons Attribution License, which permits unrestricted use, distribution, and reproduction in any medium, provided the original work is properly cited.

\begin{abstract}
In order to improve the penetration efficiency of the traditional explosively formed projectile (EFP) warhead, a new warhead with double layer liners formed collinear EFP was designed. In order to get the law of its formation and penetration into different type target, the process of the collinear EFP from warhead with double layer liners forming and penetrating into targets was simulated by using LS-DYNA software. It is obtained that the double layer liners formed two EFPs with different axial velocities under the action of detonation wave, and the velocity of the front EFP is higher than that of the rear EFP, which makes the front EFP and rear EFP separation during the flight, so it can effectively attack the reactive armor. For finite thickness target, the front EFP can effectively penetrate the hole on the target, and the rear EFP will enlarge the hole. For infinite thickness target, the collinear EFP can effectively increase the penetration depth. Compared with the single EFP, the collinear EFP from warhead with double layer liners can increase the penetration depth by $36 \%$.
\end{abstract}

Keywords: mechanics of explosion, explosively formed projectile, double layer liners, numerical simulation.

\section{Introduction}

As the explosive reactive armor and other new armor structures are widely used in tanks and other armored vehicles, the damage efficiency of the traditional explosively formed projectile (EFP) warhead has been severely challenged. Compared with the traditional EFP warhead, the collinear EFP from warhead with multi-layer liners can form multiple collinear EFP separated from the front and rear with the same energy through control. The front EFP is used to detonate the explosive reactive armor, and the rear EFP continues to penetrate the main armor, so as to effectively improve the damage efficiency to new armor. Therefore, it has become a hotspot of warhead technology research [1-7].

Fong et al. [1] carried out experimental research on the warhead with two layer and three layer spherical liners, and obtained the collinear EFP with front and rear separation. Ma et al. [2] obtained the specific process of the formation and separation of EFP with double layer liners by combining experiment and simulation. Wang et al. [3] analyzed the forming process of copper-iron composite EFP with double layer liners and the factors affecting the cladding effect. It is obtained that when the inner liner of the hemispherical structure adopts the structure with thick top and thin edge, and the outer liner adopts the structure with thin top and thick edge, a better coating effect can be achieved on the inner liner. Tosello et al. [5] carried out numerical simulation and experimental research on the underwater motion of double-layer spherical defect hood composed of tantalum and nickel. Wang et al. [6] studied a double-layer liner with copper inner cover and PTFE / Al reactive material outer cover, and carried out the penetration experiment of steel target.

The biggest advantage of collinear EFP from warhead with double layer liners is that it can be used to deal with reactive armor and increase the penetration depth. At present, the penetration 
effect of single type target is mainly analyzed, but the penetration process of two different types of target has not been studied. Therefore, in order to obtain the specific process of collinear EFP from warhead with double layer liners penetrating the finite thickness and the infinite thickness target, the process of collinear EFP from warhead with double layer liner penetrating the target was simulated by using LS-DYNA software. It provides a reference for optimizing the structural design of warhead with double layer liners which can form collinear EFP.

\section{Numerical simulation calculation and analysis}

\subsection{Numerical simulation calculation model}

In order to obtain the accurate shape parameters of different penetrators, the Lagrange algorithm was used to carry out the numerical simulation of collinear EFP with double layers forming process. The finite element model of EFP with double layer liners was established by using ANSYS / LS-DYNA software. The diameter and height of warhead are $10 \mathrm{~cm}$, and the double layer liners structure is adopted; The liners are all spherical shaped liners, in which the inner liner is close to the explosive and the outer liner is far away from the explosive; The thickness of the inner and outer liners is $0.5 \mathrm{~cm}$, and the arc radius of the inner circle is $12 \mathrm{~cm}$.

In view of the axisymmetric characteristics of projectile and target structure and load, a $1 / 4$ model was established to save calculation time. Both the explosive and the liners are made of solid 164 elements. Using Lagrange algorithm, the specific calculation model is shown in Fig. 1. The explosive is made of explosive 8701 and its density is $1.70 \mathrm{~g} / \mathrm{cm}^{3}$, and its detonation velocity is $8315 \mathrm{~m} / \mathrm{s}$; The liner is made of red copper and its density is $8.96 \mathrm{~g} / \mathrm{cm}^{3}$. In the calculation, the sliding penalty function algorithm is used between the explosive and the inner liner; Erosion contact is adopted between the inner and outer liner; A single liner defines automatic single-sided contact to simulate the contact of its outer surface after the liner is overturned, which can effectively avoid penetration and deformation between grids.

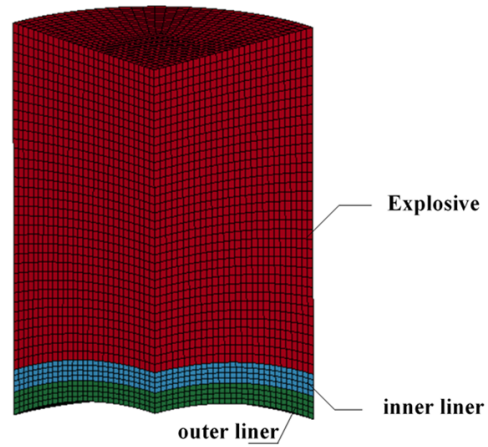

Fig. 1. The finite element model of EFP with double layer liners

Fig. 2 shows the forming process of collinear EFP from warhead with double layer liners. It can be seen from Fig. 2 that after explosive explosion, the detonation wave first acts on the inner liner and then propagates to the outer liner. At this time, the inner liner was crushed and get a certain velocity, and begins to accelerate the flight, forming the rear EFP. The energy of the shock wave further propagates and acts on the outer liner, which makes the outer liner collapse and form the front EFP. At $25 \mu \mathrm{s}$, the speed continues to increase, but gradually decreases. The velocity of the front EFP is $780 \mathrm{~m} / \mathrm{s}$ and the velocity of the rear EFP is $725 \mathrm{~m} / \mathrm{s}$. At this time, the front EFP and rear EFP have not been separated and continue to accelerate forward flight.

At $50 \mu \mathrm{s}$, the explosive completes detonation, and the detonation energy is no longer transmitted to the liner. At this time, the overall velocity of the front and rear EFP is not increasing, but due to the radial velocity difference, the front and rear EFP are still stretched and deformed 
until $630 \mu \mathrm{s}$, after which the shape of the two-stage EFP remains unchanged. Because of the velocity difference between the front EFP and rear EFP, the two stages of EFP are separated in the forming process. Fig. 3 shows the velocities of collinear EFP from warhead with double layer liners. It can be seen from Fig. 3 that the final velocity of the front EFP is $893 \mathrm{~m} / \mathrm{s}$ and that of the rear EFP is $712 \mathrm{~m} / \mathrm{s}$. The length diameter ratio of the front stage EFP is small, which is conducive to cratering when the projectile strikes the target plate, and the length diameter ratio of the rear stage EFP is long, which increases the penetration efficiency.

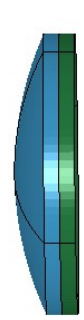

a) $0 \mu \mathrm{s}$

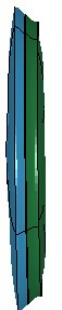

b) $25 \mu \mathrm{s}$

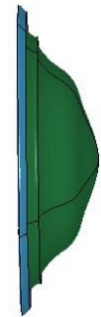

c) $50 \mu \mathrm{s}$

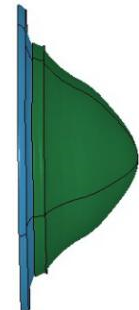

d) $75 \mu \mathrm{s}$

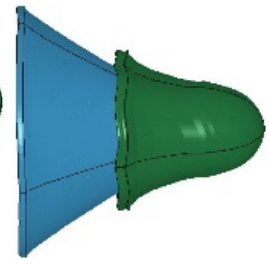

e) $250 \mu \mathrm{s}$

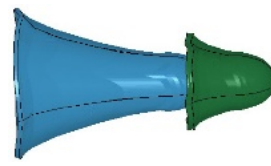

f) $630 \mu \mathrm{s}$

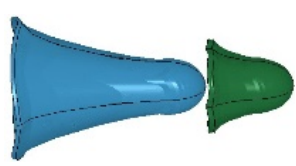

g) $770 \mu \mathrm{s}$

Fig. 2. Forming process of of collinear EFP from warhead with double layer liners

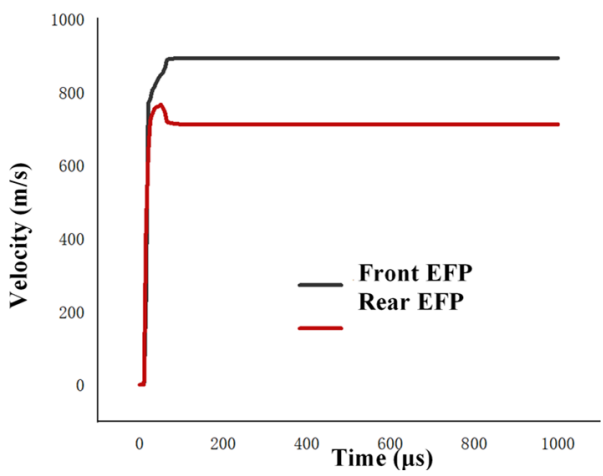

Fig. 3. Velocities of collinear EFP from warhead with double layer liners

\section{Performance analysis of penetration into steel target by EFP from warhead with double layer liners}

According to the previous analysis, it can be seen that double-layer liner can formed two stage separated EFPs, which effectively deal with the reactive armor. In order to test its performance of penetrating the reactive armor, the process of penetrating the target is numerically simulated and analyzed. Based on the model of the warhead, the simulation model of collinear EFP penetrating the $45^{\#}$ steel target is established. The contact of the front and rear EFP between the target was set in "CONTACT_ERODING_SURFACE_TO_SURFACE". Among them, the target plate is $80 \mathrm{~cm}$ away from the outer liner. Different target plate sizes are established. One size is $100 \times 100 \times 5 \mathrm{~mm}$, which simulates the finite thickness target; the other size is $100 \times 100 \times 100 \mathrm{~mm}$, which simulates the infinite thickness target.

\subsection{Penetration into the finite thickness target by collinear EFP from warhead with double layer liners}

Fig. 4 shows the process of collinear EFP penetrating the $5 \mathrm{~mm}$ thick $45^{\#}$ target. As can be seen from Fig. 4 that at $880 \mu \mathrm{s}$, the front EFP begins to penetrate the target, and at $1120 \mu \mathrm{s}$, the front EFP completes the target penetration, and at this time, the front EFP completes the opening of the target. The rear EFP continues to fly, further penetrates the target, and completes the 
reaming based on the opening of the front stage, so as to effectively enhance the armor breaking ability. At $1370 \mu \mathrm{s}$, the front and rear EFP complete the target penetration. Fig. 5 shows curve of velocity and kinetic energy of collinear EFP penetrating the $5 \mathrm{~mm}$ thick $45^{\#}$ target. It can be seen from Fig. 5 that after penetrating the target, the residual velocity of the front EFP is $691 \mathrm{~m} / \mathrm{s}$, decreased $22 \%$; The residual kinetic energy is $2.2 \mathrm{~kJ}$, attenuated by $40 \%$. The residual velocity of the rear EFP is $490 \mathrm{~m} / \mathrm{s}$, decreased by $31 \%$; the residual kinetic energy is $1.28 \mathrm{~kJ}$, attenuated by $48 \%$. In addition, it can be seen from Fig. 5 that the velocity and kinetic energy of the front EFP increase slightly after completing the process of penetrating the target. The reason is that the velocity attenuation caused by the front EFP hitting the target is less than that of the rear EFP, which leads to the rear EFP hitting the front EFP, resulting in a sudden increase in velocity and kinetic energy as shown in Fig. 5.

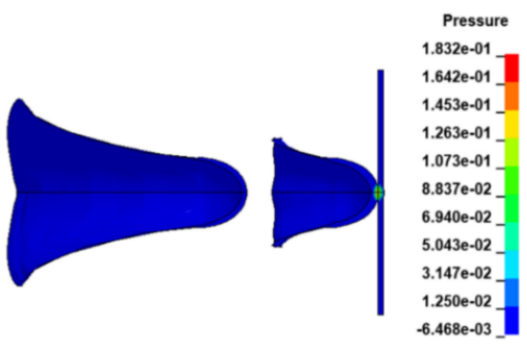

a) $t=880 \mu \mathrm{s}$

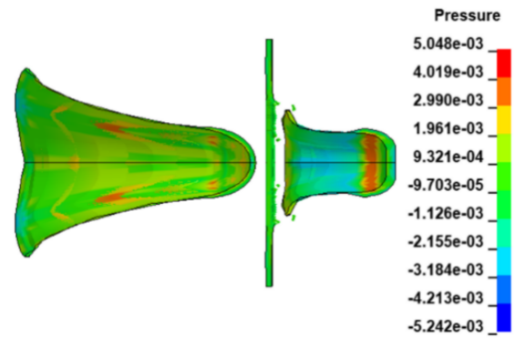

c) $t=1120 \mu \mathrm{s}$

Fig. 4. Process of collinear EFP penetrating the $5 \mathrm{~mm}$ thick $45^{\#}$ target

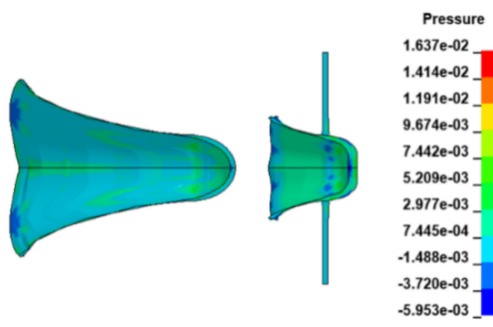

b) $t=925 \mu \mathrm{s}$

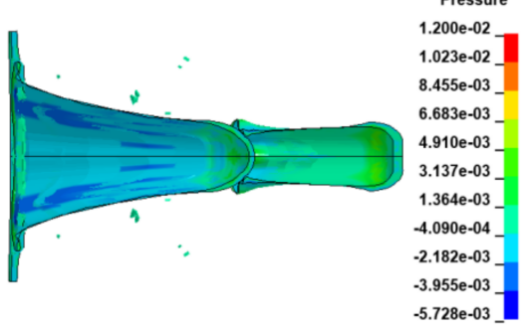

d) $t=1370 \mu \mathrm{s}$

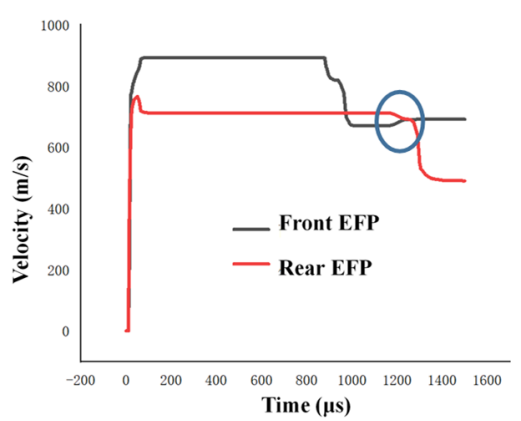

a) Velocity

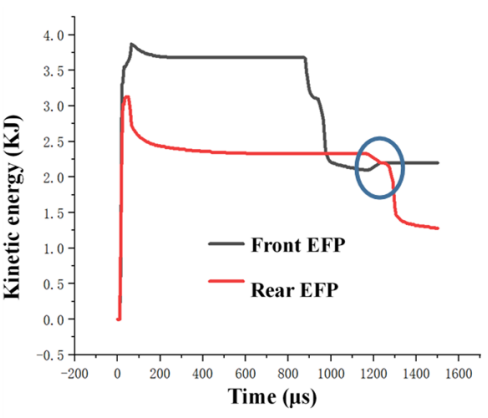

b) Kinetic energy

Fig. 5. Curve of velocity and kinetic energy of collinear EFP penetrating the $5 \mathrm{~mm}$ thick $45^{\#}$ target

\subsection{Penetration into infinite thickness target by EFP from warhead with double layer liners}

Fig. 6 shows the process of collinear EFP penetrating the $100 \mathrm{~mm}$ thick $45 \#$ target. As can be seen from Fig. 6 , at $880 \mu \mathrm{s}$, the front EFP began to penetrate the target, and the velocity and kinetic energy began to decrease. At $1060 \mu \mathrm{s}$, the remaining velocity of the front EFP is only $37 \mathrm{~m} / \mathrm{s}$, and the penetration depth reaches $33 \mathrm{~mm}$. At this time, the rear EFP starts to penetrate the target plate, 
and the rear EFP first contacts the front EFP. Due to the collision of the rear EFP, the front and rear EFP move forward together. And the projectile velocity and kinetic energy of the front EFP increase, reaching $101 \mathrm{~m} / \mathrm{s}$ at $1100 \mu \mathrm{s}$, and then there is a decay trend. At this time, the kinetic energy of the rear EFP is $1.28 \mathrm{~kJ}$, attenuated by $48 \%$, and the speed is $512 \mathrm{~m} / \mathrm{s}$, decreased by $42 \%$. Based on the penetration of the front EFP, the penetration depth of the rear EFP is $45 \mathrm{~mm}$. Compared with the single front EFP, the penetration depth of the rear EFP increases by $12 \mathrm{~mm}$ and the penetration efficiency increases by $36 \%$.
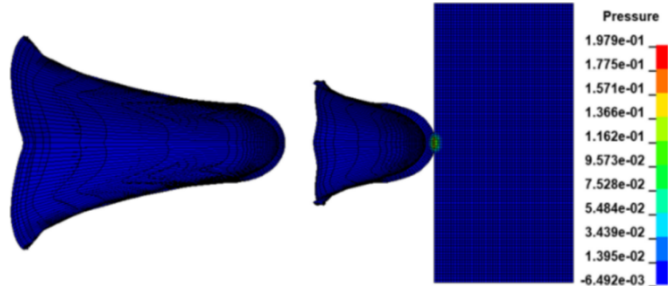

a) $t=880 \mu \mathrm{s}$

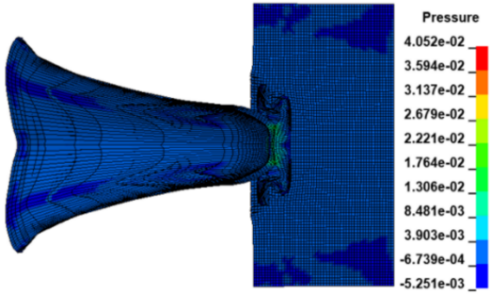

c) $t=1060 \mu \mathrm{s}$

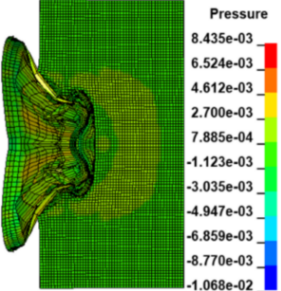

d) $t=1285 \mu \mathrm{s}$

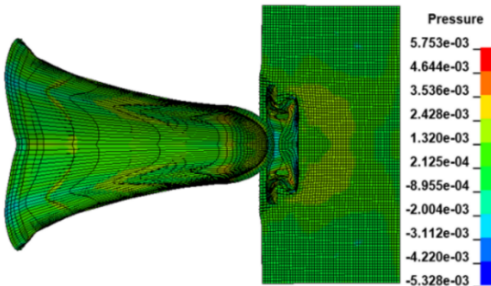

b) $t=1045 \mu \mathrm{s}$

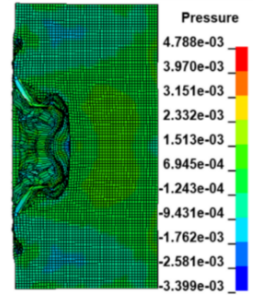

e) $t=1370 \mu \mathrm{s}$

Fig. 6. Process of collinear EFP penetrating the $100 \mathrm{~mm}$ thick $45^{\#}$ target
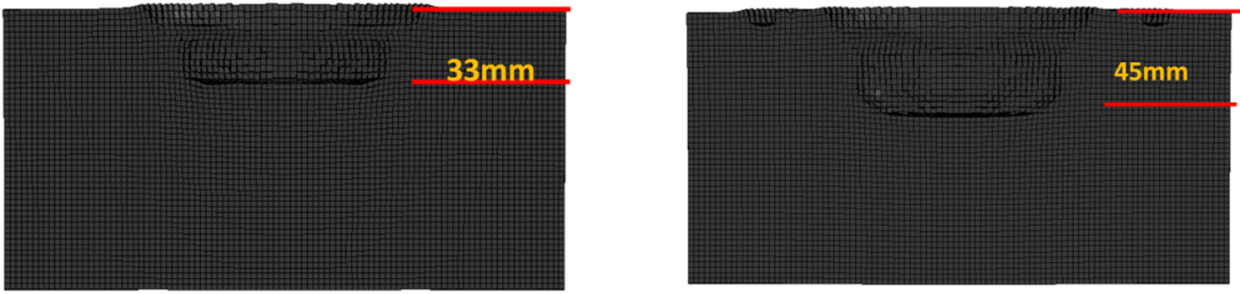

Fig. 7. Effect diagram of collinear EFP penetrating the $100 \mathrm{~mm}$ thick $45^{\#}$ target

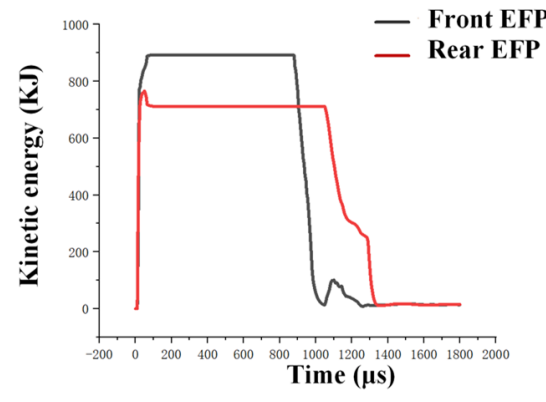

a) Velocity

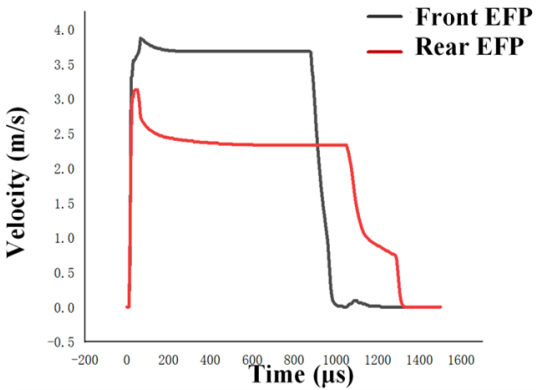

b) Kinetic energy

Fig. 8. Curve of velocity and kinetic energy of collinear EFP penetrating the $100 \mathrm{~mm}$ thick $45^{\#}$ target

\section{Conclusions}

The process of the collinear EFP from warhead with double layer liners forming and penetrating into targets was simulated by using LS-DYNA software. Through the research, the 
following results are obtained:

1) Under the action of detonation wave, the double layer liners formed two EFPs with different axial velocities. The velocity of the front EFP is higher than that of the rear EFP, which makes the front and rear separation in the flight process, so it can effectively attack the reactive armor.

2) For finite thickness target, the front EFP can effectively penetrate the hole on the target, and the rear EFP will enlarge the hole. For infinite thickness target, the collinear EFP can effectively increase the penetration depth. Compared with the single EFP, the collinear EFP from warhead with double layer liners can increase the penetration depth by $36 \%$.

\section{Acknowledgements}

This research was financially supported by the National Nature Science Foundation of China, No. 12102479.

\section{References}

[1] Fong R., Ng W., and Weiman K., "Testing and analysis of multi-liner EFP warheads," in Proceedings of the 20th International Symposium on Ballistics, 2002.

[2] H.-B. Ma, Y.-F. Zheng, H.-F. Wang, C. Ge, and C.-H. Su, "Formation and impact-induced separation of tandem EFPs," Defence Technology, Vol. 16, No. 3, pp. 668-677, Jun. 2020, https://doi.org/https://doi.org/10.1016/j.dt.2019.09.003

[3] Wang Weizhan et al., "Numerical simulation of double-layer cladding EFP molding process," (in Chinese), Journal of Missiles and Guidance, Vol. 38, No. 6, pp. 103-108, 2018.

[4] Weiman K. and Blache A., "Explosively formed projectile with tantalum penetration and steel stabilization base," in Proceedings of the 18th International Symposium on Ballistics, 1999.

[5] Tosello R., Vives M., and Tronche A., "Twin EFPs for underwater applications," in Proceedings of the18th International Symposium on Ballistics, 1999.

[6] H. Wang, H. Guo, B. Geng, Q. Yu, and Y. Zheng, "Application of PTFE/Al reactive materials for double-layered liner shaped charge," Materials, Vol. 12, No. 17, p. 2768, Aug. 2019, https://doi.org/10.3390/ma12172768

[7] Dong Liying et al., "Anti-tank mine two way EFP forming and penetration simulation," (in Chinese), Journal of Ordnance Equipment Engineering, Vol. 40, No. 10, pp. 40-44, 2019. 